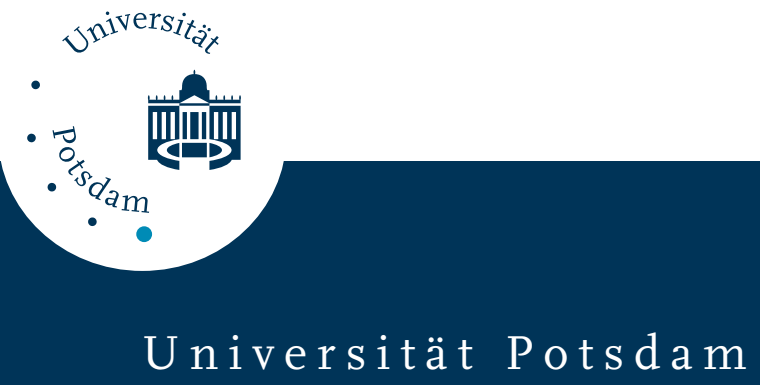

Sebastian Reich

\title{
On the local qualitative behavior of differential-algebraic equations
}

first published in:

Circuits, Systems, and Signal Processing 14 (1995), 4, p. 427-443

Postprint published at the Institutional Repository of the Potsdam University:

In: Postprints der Universität Potsdam

Mathematisch-Naturwissenschaftliche Reihe ; 159

http://opus.kobv.de/ubp/volltexte/2010/4673/

http://nbn-resolving.de/urn:nbn:de:kobv:517-opus-46739

Postprints der Universität Potsdam

Mathematisch-Naturwissenschaftliche Reihe ; 159 


\title{
ON THE LOCAL \\ QuAlitative BehaVior oF \\ DifFERENTIAL-Algebraic \\ EQUATIONS*
}

\author{
Sebastian Reich ${ }^{1}$
}

\begin{abstract}
A theoretical ramework for the investigation of the qualitative behavior of differential-algebraic equations (DAEs) near an equilibrium point is established. The key notion of our approach is the notion of regularity. A DAE is called regular locally around an equilibrium point if there is a unique vestor field such that the solutions of the DAE and the vector field are in one-to-one correspondence in a neighborhood of this equili orium point. Sufficient conditions for the regularity of an equilibrium point are stated. This in turn allows us to translate seve ral local results, as formulated for vector fields, to DAEs th at are regular locally around a g:ven equilibrium pcint (e.g. Local Stable and Unstable Manifold Theorem, Hopf theorem). It is important that these theorems are stated in terms of the given problem and not in terms of the corresponding vector field.
\end{abstract}

\section{Introduction}

Differential-algebraic equations (DAEs) are frequently identified as implicit equations

$$
F\left(x, x^{\prime}\right)=0
$$

for which $x^{\prime}$ cannot be expressed explicit y as a function of $x$. Such equations arise in many areas of science and engineerirg. In particular, constrained mechanical systems and electronic circuits may be modelled using equations of type (1) [2]. But the investigation of cortrol systems described by ordinary differential equations with certain outputs required to be identically zero can be done in terms of I)AEs as well [14]. In recent years the literature; on existence and uniqueness results has been growing rapidly (see, e.g., [2], [16], [18], [17], [3]). However, up to now, results on the qualitative behavior are available only for a few selected classes of DAEs that are characterized by restrictive conditions on the form of the mapping $F$ in (1) (see, e.g., [6], |13], [14], [21]).

* Received October 25, 1 792; revised July 22, 1993.

${ }^{1}$ Institut für Angewandte Analysis und Stochastik, Mohrenstraße 39, Berlin, O-1086, Gernany. 
In this paper we investigate the qualitative behavior of a DAE (1) near an equilibrium point $x_{0}$; i.e., a point satisfying $F\left(x_{0}, 0\right)=0$. The key notion of our approach is regularity. We call a DAE regular locally around an equilibrium point if there is a unique vector field such that the solutions of the vector field and the DAE are in one-to-one correspondence in a neighborhood of the equilibrium point under consideration. By associating to the DAE (1) a family of overdetermined DAEs, we derive in Section 3 sufficient conditions for the regularity of the DAE near an equilibrium point. Note that our approach is close to an approach taken, e.g., in [3] where the equation (1) is differentiated several times with respect to time to obtain the so-called derivative array equations. However, in contrast to the approach taken in [3], we eliminate the second order derivative $x^{\prime \prime}$ after each differentiation and thus avoid having higher order derivatives of $x$ enter into the equations. We show that this elimination step can be done by simple manipulations on matrix valued functions. In Section 4 we discuss the linearization of a nonlinear DAE in terms of the linearized DAE and the corresponding vector field. We also show in this section how the spectrum of the linearized vector field can be obtained in a direct way from the linearized DAE. These results are used in Section 5 to "translate" the Local Stable and Unstable Manifold Theorem and the Hopf theorem [10], as formulated for vector fields, to DAEs that are regular locally around the equilibrium point under consideration. It is important that these theorems are formulated in terms of the given DAE rather than in terms of the corresponding vector fields.

\section{Mathematical background}

In this section we collect some basic material needed throughout the remainder of this presentation. We begin with some standard terminology (see, e.g., [1] for details).

Throughout this presentation we consider only submanifolds of $\mathbb{R}^{n}, n>0$, which, for simplicity of exposition, are assumed to be of class $C^{\infty}$.

If $M \subseteq \mathbb{R}^{n}$ is a submanifold of $\mathbb{R}^{n}$, then $T M$ denotes the tangent bundle of $M$ and $T_{x} M$ denotes the tangent space of $M$ at $x \in M$. Now the tangent space $T_{x} M$ and the tangent bundle $T M$ can be associated canonically with a linear subspace of $\mathbb{R}^{n}$ and a submanifold of $\mathbb{R}^{n} \times \mathbb{R}^{n}$, respectively, by means of canonical embeddings $j_{x}: T_{x} M \rightarrow \mathbb{R}^{n}$, and $j: T M \rightarrow \mathbb{R}^{n} \times \mathbb{R}^{n}$. In the sequel we will not distinguish between $T_{x} M$ and $j_{x}\left(T_{x} M\right)(T M$ and $j(T M))$ and denote both spaces by $T_{x} M$ $(T M)$. Let $\mathrm{pr}_{1}: \mathbb{R}^{n} \times \mathbb{R}^{n} \rightarrow \mathbb{R}^{n}$ denote the projection of $\mathbb{R}^{n} \times \mathbb{R}^{n}$ onto the first component and let $T M \subseteq \mathbb{R}^{n} \times \mathbb{R}^{n}$ be the tangent bundle of a submanifold $M \subseteq \mathbb{R}^{n}$. Then $M=\operatorname{pr}_{1}(T M)$.

As in the case of manifolds we assume for simplicity that all the mappings under consideration will be of class $C^{\infty}$ although, once again, this condition can be reduced easily. By a vector field on a submanifold $M \subseteq \mathbb{R}^{n}$ we mean a mapping $v: M \rightarrow \mathbb{R}^{n}$ such that $v(x) \in T_{x} M$ for all $x \in M$. A mapping $x: I \rightarrow \mathbb{R}^{n}$ on the open interval $I \subseteq \mathbb{R}$ is called a solution of a given vector field $v: M \rightarrow \mathbb{R}^{n}$ 
if, for all $t \in I, x(t) \in M$ and $x^{\prime}(t)=v(x(t))$. Given a point $\left(x_{0}, p_{0}\right) \in \mathbb{P}^{n} \times \mathbb{R}^{n}$, $U\left(x_{0}, p_{0}\right) \subseteq \mathbb{R}^{n} \times \mathbb{R}^{n}$ will always clenote a sufficiently small neighbouhood of $\left(x_{0}, p_{0}\right)$ in $\mathbb{R}^{n} \times \mathbb{R}^{n}$. We will often write $U$ instead of $U\left(x_{0}, p_{0}\right)$ to further simplify notations.

In Section 3 we vrill focus on DAFis of the type

$$
G\left(x, x^{\prime}\right)=0
$$

where $G: U \rightarrow \mathbb{R}^{m}$ with $U=U\left(x_{0}, p_{0}\right) \subseteq \mathbb{R}^{n} \times \mathbb{R}^{n}, n \leq m \leq 2 n$, and $\left(x_{0}, p_{0}\right) \in G^{-1}(0)$ irı some fixed points in $\mathbb{R}^{n} \times \mathbb{R}^{n}$. For the analysis of such DAEs we will need the following results.

We call $G$ admissible (locally around $\left(x_{0}, p_{0}\right)$ ) if rank $D G\left(x_{0}, p_{0}\right)==m$ and rank $D_{p} G(x, p)=r \leq m$ is constant for all $(x, p) \in G^{-1}(0)$. The following result for admissible mappings $G$ was proven in [17]: If $G: U \rightarrow \mathbb{R}^{m}$ is adrnissible, then the sets $N=G^{-1}(0)$ and $M=\operatorname{pr}_{1}(N)$ are submanifolds of $\mathbb{R}^{n} \times \mathbb{R}^{\prime \prime}$ and $\mathbb{R}^{n}$ respectively. Furthermore, it was shown in [16] that for admissible mappings $G$ the tangent space $T_{x} M$ of $M$ at $x \in T_{x} M$ can be characterized by

$$
T_{x} M=\left\{\bar{x} \in \mathbb{R}^{n}: D_{x} G(x, p) \bar{x} \in \operatorname{rge} D_{p} G(x, p)\right\}
$$

where $(x, p) \in N$. However, to obtain a characterization of $T_{x} M$ in terms of an implicit system of equations we need the following.

Proposition 1. Let $G: U \rightarrow \mathbb{R}^{m}$ be an admissible mapping with $\operatorname{rank} D_{p} G(x, p)=$ $r$ for all $(x, p) \in G^{-1}(0)$. Then there exists a smooth mapping $S: U \rightarrow L\left(\mathbb{R}^{m}\right.$, $\left.\mathbb{R}^{m-r}\right)$ such that $S(x, p)$ has full rank for all $(x, p) \in U$ and $S(x, p) D_{p} G(x, p)=$ 0 for all $(x, p) \in N$. We call the mapping $S$ a mapping associated to $G$.

Proof. We give a constructive proof in three steps:

Step 1. There are permutations $P_{1}, P_{2} \in L\left(\mathbb{R}^{m}\right)$ such that

$$
P_{1} D_{p} G(x, p) P_{2}=V(x, p)=\left(\begin{array}{ll}
V_{11}(x, p) & V_{12}(x, p) \\
V_{21}(x, p) & V_{22}(x, p)
\end{array}\right)
$$

with $V_{11}\left(x_{0}, p_{0}\right)$ nor singular and $r \times r$.

Step 2. Define

$$
L=\left(\begin{array}{cc}
I & 0 \\
--V_{21} V_{11}^{-1} & I
\end{array}\right)
$$

where $L$ is defined for all $(x, p)$ in a st.fficiently small neighborhood $\tilde{U}$ of $\left(x_{0}, p_{0}\right)$, $\tilde{U} \subseteq U$. Let us assume, for simplicity, that $U$ was chosen such that $\tilde{U}=U$. Then we obtain on $U$

$$
L V=\left(\begin{array}{cc}
V_{11} & V_{12} \\
0 & V_{22}-V_{21} V_{11}^{-1} V_{12}
\end{array}\right) .
$$

Now, because rank $\gamma_{11}(x, p)=\operatorname{rank} D_{p} G(x, p)$ for all $(x, p) \in G^{-1}(0), V_{22}-$ $V_{21} V_{11}^{-1} V_{12}=0$ on $G^{-1}(0)$. 
Step 3. Now the mapping $S$ can be defined by

$$
S=\left(\begin{array}{ll}
-V_{21} V_{11}^{-1} & I
\end{array}\right) P_{1}
$$

and satisfies $S(x, p) D_{p} G(x, p)=S(x, p) D_{p} G(x, p) P_{2}=0$ for all $(x, p) \in$ $G^{-1}(0)$ as required.

As a consequence of Proposition 1 we obtain that $T_{x} M$ is given by

$$
T_{x} M=\operatorname{ker}\left[S(x, p) D_{x} G(x, p)\right]
$$

where $(x, p) \in N$. Note that in contrast to results in [16], the mapping $S$ in Proposition 1 is of class $C^{\infty}$ although the rank of $D_{p} G(x, p)$ is not constant on the whole set $U$ but only on $N=G^{-1}(0)$.

Remark. The construction described in the proof of Proposition 1 might not be numerically robust unless the rank of the derivatives involved is constant in a whole neighborhood of $\left(x_{0}, p_{0}\right)$. Hence our formulation in terms of constant rank conditions on submanifolds can be exploited mainly in an analytical or symbolic analysis.

Example 1. Consider the mapping $G: U \subseteq \mathbb{R}^{3} \times \mathbb{R}^{3} \rightarrow \mathbb{R}^{3}$ given by

$$
G(x, p)=\left(\begin{array}{c}
p_{3}+p_{2}-x_{1} \\
\left(x_{1}+x_{2}\right) p_{2}+x_{3} \\
x_{1}+x_{2}
\end{array}\right) .
$$

Because rank $D G(x, p)=3$ and rank $D_{p} G(x, p)=1$ for $(x, p) \in G^{-1}(0), G$ is admissible. Now, as

$$
D_{p} G(x, p)=\left(\begin{array}{ccc}
0 & 1 & 1 \\
0 & x_{1}+x_{2} & 0 \\
0 & 0 & 0
\end{array}\right)
$$

we put $P_{1}=I$ and

$$
P_{2}=\left(\begin{array}{lll}
0 & 0 & 1 \\
1 & 0 & 0 \\
0 & 1 & 0
\end{array}\right)
$$

Thus

$$
V=\left(\begin{array}{ccc}
1 & 1 & 0 \\
x_{1}+x_{2} & 0 & \\
0 & 0 & 0
\end{array}\right)
$$

and

$$
S=\left(\begin{array}{ccc}
-\left(x_{1}+x_{2}\right) & 1 & 0 \\
0 & 0 & 1
\end{array}\right)
$$

Let $G: U\left(x_{0}, p_{0}\right) \subseteq \mathbb{R}^{n} \times \mathbb{R}^{n} \rightarrow \mathbb{R}^{m}$ be an admissible mapping. Then we call the DAE

$$
G\left(x, x^{\prime}\right)=0
$$


overdetermined if $m:>n$. By a solution of a DAE (2) we mean a mapping $x$ : $I \rightarrow \mathbb{R}^{n}$ on the open interval $I \subseteq \mathbb{R}^{\prime}$ such that, for all $t \in I,\left(x(t), x^{\prime}(t)\right) \in U$ and $G\left(x(t), x^{\prime}(t)\right):=0$. Furthermore, we call the DAE regular (locally around $\left.\left(x_{0}, p_{0}\right)\right)$ if there exists a unique submanifold $W$ of $\mathbb{R}^{n}$ and a unique vector field $v: W \rightarrow \mathbb{R}^{n}$ such hat a mapping $x: I \rightarrow \mathbb{R}^{n}$ is a solution of the DAE if and only if $x$ is a solution of the vector field $v$. The vector field $v$ is then called the corresponding vector field and the manifold $W$ the configuration space.

Example 2. A pendulum with mass $m$ attached at the end of a rigid massless wire with length $l$ attached at the origin in the $\left(q_{1}, q_{2}\right)$ plane satisfies the DAE

$$
\begin{aligned}
q_{1}^{\prime} & =v_{1} \\
q_{2}^{\prime} & =v_{2} \\
m v_{1}^{\prime} & =-\lambda q_{1} \\
m v_{2}^{\prime} & =-\lambda q_{2}-m g \\
0 & =q_{1}^{2}+q_{2}^{2}-\ell^{2}
\end{aligned}
$$

where $\lambda$ is the (unkrown) tension of the wire and $g$ is the gravity constant. If we introduce the new variables $x=\left(q_{1}, q_{2}, v_{1}, v_{2}, \lambda\right) \in \mathbb{R}^{5}, p=\left(q_{1}^{\prime}, q_{2}^{\prime}, v_{1}^{\prime}, v_{2}^{\prime}, \lambda^{\prime}\right) \in$ $\mathbb{R}^{5}$, then the problem assumes the for.n (2) with

$$
G(x, p)=\left(\begin{array}{c}
p_{1}-x_{3} \\
p_{2}-x_{4} \\
m p_{3}+x_{5} x_{1} \\
m p_{4}+x_{5} x_{2}+m g \\
x_{1}^{2}+x_{2}^{2}-\ell^{2}
\end{array}\right) .
$$

On the other hand it is well known that in polar coordinates $(r, \phi)$ the motion of the pendulum is described by the second order differential equation

$$
\phi^{\prime \prime}+\frac{g}{\ell} \sin (\phi)=0 .
$$

We will see in Section 3 that (3) is the corresponding vector field of the above DAE written as a second order differential equation.

Let us finally introduce the following notations. Let $v^{l}: M \rightarrow \mathbb{R}^{n}$ be a linear vector field on a linear subspace $M$ of $\mathbb{R}^{n}$; i.e., $v^{l} \in L\left(M, \mathbb{R}^{n}\right)$. Then the spectrum of $v^{l}$, denoted by $\rho\left(v^{l}\right)$, is the set of all $\mu \in \mathbb{C}$ such that there exists a $y \in M$, $y \neq 0$, with

$$
\left(\mu j-v^{l}\right) y=0
$$

where $j: M \rightarrow \mathbb{R}^{n}$ is the canonical embedding of $M$ into $\mathbb{R}^{n}$. Consider now the matrix pencil $(A, B)[8]$ with $A, B \in L\left(\mathbb{R}^{n}\right)$. Then the set of all $\mu \in \mathbb{C}$ satisfying $\operatorname{det}(\mu A+B)=0$, denoted by $\rho(A, B)$, is called the spectrum of the matrix pencil. 


\section{Existence and uniqueness of solutions near an equilibrium point}

Throughout this paper we consider DAEs

$$
F\left(x, x^{\prime}\right)=0
$$

$F: \mathbb{R}^{n} \times \mathbb{R}^{n} \rightarrow \mathbb{R}^{n}$, with an equilibrium point $x_{0} \in \mathbb{R}^{n}$; i.e., $F\left(x_{0}, 0\right)=0$. We assume that

$$
\text { A1 : } \operatorname{rank} D_{x} F\left(x_{0}, 0\right)=n,
$$

which implies that $x_{0}$ is an isolated equilibrium point of (4), and that

$$
\text { A2 : } \operatorname{rank} D_{p} F(x, p)=\text { const }
$$

in a neighborhood of $\left(x_{0}, 0\right)$ in $F^{-1}(0)$. Both assumptions together imply that the mapping $F$ is admissible locally around $\left(x_{0}, 0\right)$.

In this section we derive results on the existence and uniqueness of solutions near the equilibrium point $x_{0}$, although most of the results of this section can be easily generalized to arbitrary points $(x, p) \in F^{-1}(0)$. For that reason we consider (4) only on a sufficiently small neighborhood $U=U(x, 0,0)$ of the equilibrium point $\left(x_{0}, 0\right)$. In contrast to other approaches on the existence and uniqueness of solutions (see, e.g., [3], [17]) our results are based on a family of overdetermined DAEs which we define as follows:

Definition 1. Let a DAE (4) with an equilibrium point $x_{0}$ be given. Assume that assumptions $\mathrm{A} 1$ and $\mathrm{A} 2$ hold. Let $U$ be a sufficiently small neighborhood of $\left(x_{0}, 0\right)$. Then we define the family $\left(G_{i}\right)$ of mappings $G_{i}: U \rightarrow \mathbb{R}^{m_{i}}$ by:

$$
\begin{aligned}
G_{0}(x, p) & :=F(x, p) \\
G_{i+1}(x, p) & :=\left(\begin{array}{c}
G_{0}(x, p) \\
S_{i}(x, p) D_{x} G_{i}(x, p) p
\end{array}\right) \quad(i=1, \ldots, s-1)
\end{aligned}
$$

where $S_{i}$ is a mapping associated to $G_{i}$ (see Proposition 1) and $s$ is the largest integer such that the $G_{i}$ 's are admissible and $G_{s}^{-1}(0) \neq G_{s-1}^{-1}(0)$. We say that the DAE (4) is of degree $s$ locally around the equilibrium point $x_{0}$.

Example 3. The DAE associated to the mapping $G$, as considered in Example 1, is of degree one. The mapping $G_{1}$ is given by

$$
G_{1}(x, p)=\left(\begin{array}{c}
G(x, p) \\
\left(x_{1}+x_{2}\right) p_{1}+p_{2}\left(p_{1}+p_{2}\right)+p_{3} \\
p_{1}+p_{2}
\end{array}\right)
$$

and $G_{2}=G_{1}$ because rank $D_{p} G_{1}(x, p)=3$. 
Example 4. Let us :onsider quasilinıar DAEs

$$
F\left(x, x^{\prime}\right):=A(x) x^{\prime}+f(x)=0
$$

i.e., DAEs for which $x^{\prime}$ enters linearly into the equations. Let us assume that rank $A(x)=k$ for all $x \in E$, where $V$ is a sufficiently small neighborhood of $x_{0}$ in $\mathbb{R}^{n}$, $f\left(x_{0}\right)=0$, and rank: $D f\left(x_{0}\right)=n$. In this case $F$ is admissible and we simply have $D_{p} F=A$. If $S$ is a mapping associated to $F$; i.e., $S(x) A(x)=0$, then ve obtain

$$
M=\left\{x \in \mathbb{R}^{n}: g(x):=S(x) f(x)=0\right\}
$$

and

$$
T_{x} M:=\operatorname{ker} \operatorname{Dg}(x) .
$$

Thus the mapping $G_{1}$ in Definition 1 can be modified to

$$
G_{1}(x, p)=\left(\begin{array}{c}
A(x) p+f(x) \\
D g(x) p
\end{array}\right)
$$

which is again linear in the variable $p$ and, consequently, similar modifications can be made for all mappings $G_{i}$.

In the following proposition we tise the special structure of the DAE' (4) near $x_{0}$ to state sufficient: conditions for the admissibility of the mappings $G_{i}$.

Proposition 2. Assume that $G_{i-1}$ is admissible. Then $G_{i}$ is admissibie if rank $D_{p} G_{i}(x, p)=$ consit. for all $(x, p) \in: G_{i}^{-1}(0)$.

Proof. We have to show that the adm ssibility of $G_{i-1}$ implies that $D G_{i}\left(x_{0}, 0\right)$ has full rank. By definition (and using the fact that $p_{0}=0$ )

$$
D G_{i}\left(x_{0}, \jmath\right)=\left(\begin{array}{cc}
D_{x} G_{0}\left(x_{0}, 0\right) & D_{p} G_{0}\left(x_{0}, 0\right) \\
0 & S_{i-1}\left(x_{0}, 0\right) D_{x} G_{i-1}\left(x_{0}, 0\right)
\end{array}\right) .
$$

Now rank $D_{x} G_{0}\left(x_{\mathrm{c}}, 0\right)=n$ by assumption $\mathrm{A} 1$ and $S_{i-1}\left(x_{0}, 0\right) D_{x} G_{i-1}\left(x_{0}, 0\right)$ has full rank by the admissibility of $G_{i-1}$.

We associate now with each of the mappings $G_{i}$ a DAE of type (2). Comparing their solution behavior we obtain

Proposition 3. Let (4) be a DAE of degree s locally around $x_{0}$, then the DAEs

$$
G_{i}\left(x, x^{\prime}\right)=0 \quad(i=0, \ldots, s)
$$

have identical soluition behavior. 
Proof. Let us introduce the sets $N_{i}=G_{i}^{-1}(0), M_{i}=\operatorname{pr}_{1}\left(N_{i}\right)$. By the admissibility of the mappings $G_{i}$, the sets $N_{i}, M_{i}$ are manifolds and satisfy $N_{i+1} \subseteq N_{i}, M_{i+1} \subseteq$ $M_{i}$. Thus, by the definition of the mappings $G_{i}$ :

$$
N_{i+1}=\bigcup_{x \in M_{i}}\left[N_{0} \cap\left\{x \times T_{x} M_{i}\right\}\right]
$$

and thus $N_{i+1}=N_{0} \cap T M_{i}$. Now because of $N_{0} \cap T M_{i}=N_{0} \cap T M_{i-1} \cap T M_{i}=$ $N_{i} \cap T M_{i}$, we obtain $N_{i+1}=N_{i} \cap T M_{i}$. As already shown in [18], a mapping $x: I \rightarrow \mathbb{R}^{n}$ satisfies $\left(x(t), x^{\prime}(t)\right) \in N_{i}$ if and only if $\left(x(t), x^{\prime}(t)\right) \in N_{i} \cap T M_{i}$.

Remark. Our approach is close to an approach taken, e.g., in [3] where the equation (4) is differentiated several times with respect to $t$ to obtain the so-called derivative array equations. However, in contrast to the approach taken in [3], we eliminate the second order derivative $x^{\prime \prime}$ after each differentiation and thus avoid having higher order derivatives of $x$ enter into the equations. The advantage of the approach taken in [3] is that it does not involve constant rank conditions in the intermediate steps.

We are now ready to state sufficient conditions for the regularity of a DAE (4) locally around an equilibrium point $x_{0}$.

Theorem 1. Let (4) be a DAE of degree s locally around $\left(x_{0}, 0\right)$. If

$$
\operatorname{rank} D_{p} G_{s}\left(x_{0}, 0\right)=n
$$

then (4) is regular locally around $\left(x_{0}, 0\right)$. The corresponding vector field $v: W \rightarrow$ $\mathbb{R}^{n}$ is characterized by

$$
\text { graph } v=G_{s}^{-1}(0) \text {. }
$$

Proof. Rank $D_{p} G_{s}\left(x_{0}, 0\right)=n$ implies that $N_{s}=G_{s}^{-1}(0)$ is the graph of a function $v: M_{s} \rightarrow \mathbb{R}^{n}$. Because $N_{s} \cap T M_{s}=N_{s}, v(x) \in T_{x} M_{s}$ for all $x \in M_{s}$. Thus Proposition 3 implies the regularity of (4) locally around $\left(x_{0}, 0\right)$.

Definition 2. In correspondence with the definitions given, e.g., in [2], [17], we call a DAE (4) of index s locally around $\left(x_{0}, 0\right)$ if the conditions of Theorem 1 are satisfied.

Example 5. The DAE considered in Example 2 is of index three. The DAE corresponding to the mapping $G_{3}$ can be rewritten as 


$$
\begin{aligned}
q_{1}^{\prime} & =v_{1} \\
q_{2}^{\prime} & =v_{2} \\
m v_{1}^{\prime} & =-\lambda q_{1} \\
m v_{2}^{\prime} & =-\lambda q_{2}-m \xi^{\prime} \\
\lambda^{\prime} & =\frac{1}{l^{2}}\left(3 m v_{2} g+2 v_{1} q_{1} \lambda+2 v_{2} q_{2} \lambda\right) \\
0 & =q_{1}^{2}+q_{2}^{2}-l^{2} \\
0 & =q_{1} v_{1}+q_{2} v_{2} \\
0 & =\frac{l^{2}}{m} \lambda+q_{2} g-\left(v_{1}^{2}+v_{2}^{2}\right) .
\end{aligned}
$$

Introducing polar coordinates $(r, \phi)$ in the $\left(q_{1}, q_{2}\right)$-plane and denoting $r^{\prime}$ and $\phi^{\prime}$ by $\rho, \theta$, respectively, this DAE reduces to (after elimination of the variable $\lambda$ )

$$
\begin{aligned}
\phi^{\prime} & =\theta \\
r^{\prime} & =\rho \\
l \theta^{\prime} & =-g \sin (\phi) \\
\rho^{\prime} & =0 \\
0 & =r^{2}-l^{2} \\
0 & =r \rho
\end{aligned}
$$

and the resulting ODE

$$
\begin{aligned}
\phi^{\prime} & =\theta \\
\theta^{\prime} & =-\frac{g}{l} \sin (\phi)
\end{aligned}
$$

represents the corresponding vector field of the DAE on the manifold $M=\{(q, v)$ : $\left.q_{1}^{2}+q_{2}^{2}=r^{2}=l^{2}, q_{1} p_{1}+q_{2} p_{2}=r \rho=0\right\}$.

\section{Linearization of a DAE in an equilibrium point}

Many local qualitative results for vector fields are based on the linearization of a vector field $v: M \rightarrow \mathbb{R}^{n}$ along a given solution $x: I \rightarrow \mathbb{R}^{n}$. In the sequel we focus on problems where this solution is an equilibrium point $x_{0} \in M^{\prime}$; i.e., $v\left(x_{0}\right)=0$. The investig,ation of the resulting linear vector field $v^{l} \in L\left(T_{x_{0}} M, \mathbb{R}^{n}\right)$, given by

$$
v^{l}:=D v\left(x_{0}\right),
$$

allows us in many cases to determine the qualitative solution properties of the vector field $v$ in a sufficiently small neighborhood of the equilibrium point. [10]. Of course one would like to apply this strategy to regular DAEs as well. For that reason let $x_{0} \in \mathbb{R}^{n}$ be an equilibrium point of the DAE (4); i.e., assume that

$$
0=F\left(x_{0}, 0\right) \text {. }
$$


Then the linearization of the DAE (4) along the solution $x(t)=x_{0}$ results in the linear DAE

$$
A \bar{x}^{\prime}+B \bar{x}=0
$$

where $A=D_{p} F\left(x_{0}, 0\right)$ and $B=D_{x} F\left(x_{0}, 0\right)$.

It seems reasonable to conjecture that, whenever the DAE (4) is regular locally around an equilibrium point $x_{0}$, the linearized DAE (5) is regular too and that the corresponding vector field of (5) is given by the linearization of the corresponding vector field of (4) at $x_{0}$. However, as shown by Example 6, this conjecture does not always hold.

Example 6. Consider the DAE

$$
x x^{\prime}=-x^{2}
$$

$x \in \mathbb{R}$. Note that this DAE does not satisfy assumptions A1 and A2 of Section 3 at $(0,0)$ and that therefore no degree can be defined for the DAE locally around $(0,0)$. However, the DAE is regular locally around $(0,0)$ and the corresponding vector field is given by $x^{\prime}=-x$. Now, this is already a linear differential equation and we expect linearization of $(6)$ at $(0,0)$ to yield a regular DAE with $x^{\prime}=-x$ as the corresponding vector field. However, what we obtain really is the "DAE" $0=0$, which is obviously nonregular. Note that this situation can never occur for a DAE (4) that satisfies assumption A1.

Assumption A1 implies that the linearized DAE (5) is regular whether or not the given DAE (4) is regular locally around the equilibrium. But even if the DAE (4) is regular this does not imply that the corresponding vector field of the linear DAE (5) is equivalent to the linearization of the corresponding vector field of the nonlinear problem at $x_{0}$.

Example 7. Consider the DAE

$$
\begin{aligned}
x^{\prime} & =-y \\
0 & =y^{3}-x
\end{aligned}
$$

with $(x, y) \in \mathbb{R} \times \mathbb{R}$. This DAE satisfies assumptions A1 and A2 but does not satisfy the conditions of Theorem 1 . However, because $(x(t), y(t))=(0,0)$ is the only solution with $(x(0), y(0))=(0,0)$, the corresponding vector field of $(7)$ is given by

$$
\left(x^{\prime}, y^{\prime}\right)= \begin{cases}(-y,-1 / 3 y) & \text { for }(x, y) \in M_{1} \backslash\{(0,0)\} \\ (0,0) & \text { for }(x, y)=(0,0)\end{cases}
$$

Because this vector field has a discontinuity at the equilibrium point $(0,0)$ no linearization of the vector field at the equilibrium point exists. However, linearization of the DAE $(7)$ at $(0,0)$ yields the linear DAE

$$
\begin{aligned}
\bar{x}^{\prime} & =\bar{y} \\
0 & =\bar{x}
\end{aligned}
$$


which is of index two and possesses only the trivial solution $(\bar{x}(t), \bar{y}(t))=(0,0)$.

In the following theorem we state sufficient conditions under which the linearized DAE (5) properly reflects the linearized solution behavior of the given nonlinear problem (4).

Theorem 2. Let (4) be a DAE of index s locally around an equilibrium point $x_{0}$. Assume that the DAE satisfies assumption A1. Denote the corresponding vecior field of (4) by $v: M \rightarrow \mathbb{R}^{n}$ and the corresponding vector field of the linearized $D A E(5)$ by $v^{l}: M^{l} \rightarrow \mathbb{R}^{\prime \prime}$. Then we have

$$
D v\left(x_{0}\right)=v^{l}
$$

where $D v\left(x_{0}\right) \in L\left(T_{x_{0}} M, \mathbb{R}^{n}\right)$ is the linearization of $v$ at $x_{0}$ and $M^{l}=T_{x_{0}} M$.

Proof. As in the proof of Proposition 3 we introduce the sets $N_{i}=G_{i}^{-1}(0)$, $M_{i}=\operatorname{pr}_{1}\left(N_{i}\right), i=0, \ldots, s$. We denote the corresponding sets of the linearized DAE by $N_{i}^{l}, M_{i}^{l}$, respectively. We prove the theorem by induction. By definition we have $N_{0}^{l}=T_{\left(x_{0}, 0\right)} N_{())}$. Let us assume that $N_{i}^{l}=T_{\left(x_{0}, 0\right)} N_{i}$ holds for somie $i$, $s>i \geq 0$. Then $M_{i}^{l}=T_{x_{0}} M_{i}$ and $T M_{i}^{l}==T_{\left(x_{0}, 0\right)} T M_{i}$ as well. Now, as show $\mathrm{n}$ in the proof of Proposition 3, we have

$$
N_{i+1}=N_{i} \cap T M_{i}
$$

which implies that

$$
T_{\left(x_{0}, 0\right)} N_{i+1}=T_{(j: 0,0)}\left(N_{i} \cap T M_{i}\right) .
$$

Now the admissibility of the mapping $G_{i}$ gives

$$
\begin{aligned}
\Gamma_{\left(x_{0}, 0\right)} N_{i+1} & =T_{\left(x_{0}, 0\right)} N_{i} \cap T_{\left(x_{0}, 0\right)} T M_{i} \\
& \left.=N_{i}^{l}\right\urcorner T M_{i}^{l} \\
& =N_{i+1}^{l} .
\end{aligned}
$$

Finally we observe that graph $v=N_{s}$ and graph $v^{l}=N_{s}^{l}$.

In the following proposition we show that the regularity of the linearized DAE simplifies the conditions for the regularity of the nonlinear problem as stated in Theorem 1.

Proposition 4. Let $x_{0}$ be an equilibrium point of a DAE (4) and let (5) be the linearization of (4) in $x_{0}$. Assume that (5) is of index $s$ and that the DAE (4) is at least of degree $s-1$ locally around $x_{10}$. Then (4) is of index s locally aro and the equilibrium point $x_{0}$.

Proof. We use the same notation as in the proof of Theorem 2. Because (4) is at least of degree $s-1, G_{s-1}$ is defined and admissible. Thus $G_{s}$ is defined too and, as shown in the proof of Proposition 2, $D G_{s}\left(x_{0}, 0\right)$ has full rank. Therefore $N_{s}=$ $N_{s-1} \cap T M_{s-1}$ is a manifold and $T_{\left(x_{0}, 0\right)} N_{s}=N_{s}^{l}$. This implies rank $D_{p} G_{s}\left(x_{0}, 0\right)=$ $n$. Thus $G_{s}$ is admissible and satisfies the conditions of Theorem 1 . 
Example 8. Let $x_{0}$ be an equilibrium point of a quasilinear DAE

$$
F\left(x, x^{\prime}\right)=A(x) x^{\prime}+f(x)=0 .
$$

Assume that rank $A(x)=k$ for all $x \in V$, where $V$ is a sufficiently small neighborhood of $x_{0}$ in $\mathbb{R}^{n}$, and that rank $D f\left(x_{0}\right)=n$. This implies that $F$ is admissible. Furthermore, $F_{p}=A$. Let $S(x)$ be the mapping associated to $F$ and define the mapping $g: V \rightarrow \mathbb{R}^{n-k}$ by $g(x)=S(x) f(x)$. Then $N_{0}$ and $M_{0}$ are given by

$$
\begin{aligned}
& N_{0}=\left\{(x, p) \in V \times \mathbb{R}^{n}: A(x) p=f(x)\right\} \\
& M_{0}=\{x \in V: g(x)=0\} .
\end{aligned}
$$

If now the linearized DAE is of index one, then Proposition 4 implies that the DAE is of index one locally around the equilibrium point $x_{0}$. More generally, if

$$
\operatorname{rank}\left(\begin{array}{c}
A(x) \\
D g(x)
\end{array}\right)=\text { const. }
$$

for all $x \in V$, then the mapping $G_{1}$ given by

$$
G_{1}(x, p)=\left(\begin{array}{c}
A(x) p+f(x) \\
D g(x) p
\end{array}\right)
$$

is admissible too. Thus, if the linearized DAE is of index two, then Proposition 4 yields in this case that the DAE is of index two locally around the equilibrium point $x_{0}$.

It is well known that the spectrum $\rho\left(v^{l}\right)$ of a linear vector field $v^{l}$ plays a crucial role in computing the solution behavior. If $v^{l}$ is the corresponding vector field of a linear DAE (5), then, as shown in the next proposition, the spectrum $\rho\left(v^{l}\right)$ can be obtained directly from the matrix pencil $(A, B)$ of the linear DAE.

Proposition 5. Let (5) be a DAE of index $s$ and let $v^{l}: M^{l} \rightarrow \mathbb{R}^{n}$ be the corresponding vector field of this DAE. Then the spectrum of $v^{l}$ and the spectrum of $(A, B)$ satisfy

$$
\rho\left(v^{l}\right)=\rho(A, B) .
$$

Proof. If $\mu \in \rho\left(v^{l}\right)$ then there is a $y \in M^{l}, y \neq 0$, such that

$$
\left(\mu j-v^{l}\right) y=0 \text {. }
$$

Furthermore, by definition of the corresponding vector field we have

$$
\left(A v^{l}+B\right) y=0
$$

for all $y \in M^{l}$. Thus, if $\mu \in \rho\left(v^{l}\right)$ then $\mu \in \rho(A, B)$ because of

$$
(\mu A+B) y=0 .
$$

On the other hand, if $\mu \in \rho(A, B)$, then the corresponding $y \in \mathbb{R}^{n}$ in (10) has to satisfy $y \in M^{l}$. This implies $\rho\left(v^{l}\right)=\rho(A, B)$. 


\section{Applications}

The preceding results, especially Theorem 1, Theorem 2, and Proposition 5, can be used to discuss the solution behavior of DAEs near an equilibrium point $x_{0} \in \mathbb{F}^{n}$. According to the results of Section 4 one can hope that the linearized DAE plays a crucial role in determining the qualitative behavior of (4) near an equilibrium point $x_{0}$. For that reason let (5) be the linearization of (4) at $x_{0}$ and assume that (5) is regular. Then, according to Proposition 5 the solution properties of the linear DAE (5) can be obtained from the spectrum $\rho(A, B)$ with

$$
\begin{aligned}
& \rho^{\prime \prime}:=\{\mu \in \rho(A, B): \operatorname{Re} \mu<0\} \\
& \rho^{z}:=\{\mu \in \rho(A, B): \operatorname{Re} \mu=0\} \\
& \rho^{u}:=\{\mu \in \rho(A, B): \operatorname{Re} \mu>0\} .
\end{aligned}
$$

Especially, then are invariant subspaces of the DAE (5) associated to $\rho^{*}, \star=$ $s, c, u$, call these $X^{*}$, and $M^{l}=X^{s} \oplus X^{c} \oplus X^{u}$ where $M^{l}$ is the configuration space of the DAE (5). The association is that $X^{*}$ is spanned by the eigenvertors $v_{i} \in \mathbb{R}^{n}$ corresponding to the eigenvalues $\mu_{i} \in \rho^{*}$, i.e.,

$$
\left(\mu_{i} A+B\right) v_{i}=0 .
$$

Any solution $\bar{x}$ of (5) with $\bar{x}(0) \in X^{s}$ terids exponentially to 0 as $t \rightarrow \infty$ and any solution with $\bar{x}(0) \in X^{u}$ to 0 as $t \rightarrow-\infty$. As long as the eigenvalues of $\rho^{c}$ are simple, the solutions with $\bar{x}(0) \in X^{c}$ are periodic.

We turn now to the nonlinear problem (4) and assume that the conditions of Theorem 1 are satisfied. Then, in accordance with corresponding definitions for vector fields, we call $x_{0}$ a hyperbolic equilibrium point if the linearized DAE satisfies $\rho^{c}=\emptyset$. Furth ermore, let $x\left(\cdot ; j_{a}\right): I \rightarrow \mathbb{R}^{n}$ denote solutions of (4) in a sufficiently small neighborhood $U$ of $\left(x_{0}, 0\right)$ with $x\left(0, x_{a}\right)=x_{a}$, then we define [10]

$$
\begin{aligned}
W^{s}:= & \left\{x_{a} \in \mathbb{R}^{n}: \exists x\left(\cdot ; x_{a}\right):[0, \infty) \rightarrow \mathbb{R}^{n}\right. \\
& \text { suchthat } \left.x\left(t ; x_{a}\right) \rightarrow x_{0} \text { for } t \rightarrow \infty\right\} \\
W^{u}:= & \left\{x_{a} \in \mathbb{R}^{n}: \exists x\left(\cdot ; x_{a}\right):[0,-\infty) \rightarrow \mathbb{R}^{n}\right. \\
& \text { suchthat } \left.x\left(t ; x_{a}\right) \rightarrow x_{0} \text { for } t \rightarrow-\infty\right\} .
\end{aligned}
$$

For hyperbolic equil brium points the solutions of (5) resemble the solution behavior of (4) in a sufficiently small neighborhood of $x_{0}$. Specifically:

Theorem 3 (Local Stable and Unstable Manifold). Let $x_{0}$ be an equilibrium point of the DAE (4). Assume that (4) is of index $s, s \geq 1$, locally around $x_{0}$ and that $x_{0}$ is a hyperbolic equilibrium point. Consider the DAE (4) on a sufficiently small neighborhooc! $U$ of $\left(x_{0}, 0\right)$. Then the sets $W^{s}$ and $W^{u}$ are submarifolds of the configuration space $W$ that are tangent to $X^{s}, X^{u}$, respectively, ai $x_{0}$; i.e., $T_{x_{0}} W^{s}=X^{s}$ and $T_{: c_{0}} W^{u}=X^{u}$. 
Proof. By assumption the DAE (4) is regular on $U$. Furthermore, by Theorem 2 and Proposition 5, the point $x_{0}$ is a hyperbolic equilibrium point of the corresponding vector field. Thus one can apply the stable and unstable manifold theorem as formulated for vector fields in [10] to the DAE (4).

Remarks. The set $W^{s}\left(W^{u}\right)$ is called the local stable (unstable) manifold. These manifolds can be used to give a coordinate system on the configuration space in a neighborhood of $x_{0}$. Furthermore, if $\rho^{u}=\emptyset$, then the equilibrium point $x_{0}$ is asymptotically stable in the sense of Lyapunov [10].

Example 9. A Lagrangian formulation of the equations describing a constrained multibody system may be written as

$$
\begin{aligned}
q^{\prime} & =v \\
M(q) v^{\prime} & =f(q, v)-G^{T}(q) \lambda \\
0 & =g(q)
\end{aligned}
$$

where $M(q)$ is a regular (symmetric, positive definite) mass matrix, $f$ is a vector of applied forces, and $\lambda$ represents the Lagrange multipliers or constraint forces coupled to the system by the constraint matrix $G(q):=D g(q)$, which is assumed to have full rank. This implies that the above DAE is of index three. Let us assume that $f\left(q_{0}, 0\right)=0$, then the point $\left(q_{0}, 0\right)$ is an equilibrium solution of the corresponding mechanical systems. To determine the stability properties of $\left(q_{0}, 0\right)$ we can make use of Theorem 3. Linearization yields the following linear DAE:

$$
\left(\begin{array}{ccc}
I & 0 & 0 \\
0 & M\left(q_{0}\right) & 0 \\
0 & 0 & 0
\end{array}\right)\left(\begin{array}{c}
\bar{q}^{\prime} \\
\bar{v}^{\prime} \\
\bar{\lambda}^{\prime}
\end{array}\right)=\left(\begin{array}{ccc}
0 & I & 0 \\
D_{p} f\left(q_{0}, 0\right) & D_{v} f\left(q_{0}, 0\right) & -G^{T}\left(q_{0}\right) \\
G\left(q_{0}\right) & 0 & 0
\end{array}\right)\left(\begin{array}{c}
\bar{q} \\
\bar{v} \\
\bar{\lambda}
\end{array}\right) .
$$

From this one gets the matrices $A$ and $B$ and finally one has to compute the generalized eigenvalues of the matrix pencil $(A, B)[7]$.

The study of fixed points becomes more delicate when they are not hyperbolic. Nonhyperbolic fixed points often arise in bifurcation problems. Here we restrict ourselves to the case in which $\rho^{c}$ contains two simple eigenvalues $\pm j \omega$ on the imaginary axis. For vector fields the resulting bifurcation is called the Hopf bifurcation [10], [11]. A first result on Hopf bifurcations for DAEs of index one can be found in [21].

Let

$$
F\left(x, x^{\prime} ; \lambda\right)=0 \quad(\lambda \in \mathbb{R})
$$

be a one-parametric family of DAEs with an equilibrium point $x_{0}$ for $\lambda=0$. Assume that assumption $\mathrm{A} 1$ holds for $\lambda=0$. Then the implicit function theorem implies that there are neighborhoods $\Lambda \subseteq \mathbb{R}$ and $V \subseteq \mathbb{R}^{n}$ of 0 and $x_{0}$ such that the system of equations

$$
F(x, 0 ; \lambda)=0
$$


has exactly one solution $x \in V$ fo: all $\lambda \in \Lambda$; i.e., there is a one-parametric family $x_{0}(\lambda)$ of equilibrium points with $x_{0}(0)=x_{0}$.

Assume that (11) is of index $s$ locally around $x_{0}(\lambda)$ for all $\lambda \in \Lambda$ and that the mapping $G_{s}(., . ; \lambda)$ depends smcothly on $\lambda$. Then the corresponding vector field $v(. ; \lambda)$ also depend smoothly on $\lambda$. Furthermore, the linearized DAEs

$$
A(\lambda) \bar{x}^{\prime}+B(\lambda) \bar{x}=0 \quad(\lambda \in \Lambda)
$$

with $A(\lambda):=D_{p} F\left(x_{0}(\lambda), 0 ; \lambda\right)$ and $B(\lambda):=D_{x} F\left(x_{0}(\lambda), 0 ; \lambda\right)$ are regular. Assume now that the spectrum of the matrix pencll $(A(\lambda), B(\lambda)$ ) contains exactly one pair of complex eigenvalues $\mu_{1,2}=\rho(\lambda) \pm j \omega(\lambda)$ such that

$$
\text { A3 : } \quad \rho(0)=0, \omega(0) \neq 0
$$

and that (12) has no other eigenvalues on the imaginary axis for $\lambda=0$. This implies that, for $\lambda=0$, the linearized DAE satisfies $\rho^{c}=\{ \pm \omega(0)\}$ and that the equilibrium points $x_{0}(\lambda)$ are hyperbolic for $\lambda: \neq 0$. Using results in [10], a Hopf theorem for DAEs of the above type follows easily. Specifically:

Theorem 4 (Hopf). Let us consider the one-parametric family of DAEs (11) where $x_{0}$ is an equilibrium point of (11) for $\lambda=0$ satisfying assumption A1. Assume that (11) is of index s locally around $x_{0}(\lambda)$ for all $\lambda \in \Lambda$ and that the mapping $G_{s}(., . ; \lambda)$ depends smoothly on $\lambda$. Let us assume furthermore that assumption $A 3$ holds. Then, in any neighborhood $U$ of $x_{0}$ in $\mathbb{P}^{n}$ and any given $\lambda_{0}$ there is a $\bar{\lambda}$ with $|\bar{\lambda}|<\lambda_{0}$ such that (11) witr: $\lambda=\bar{\lambda}$ has a nontrivial periodic solution in $U$.

Remark. The theorem in ts above formulation does not allow us to draw any conclusion on whether the periodic solution is locally unique or not. To obtain such results we would need a theory of normal forms for DAEs. First results in that direction can be founci in [5].

As an application of Theorem 4, let us consider the numerical computation of Hopf points for DAEs in terms of an augmented system of nonlinear equations. This so-called direct approach has been very successfully applied in the OJE context [20]. Here we give a brief outline on how to generalize these results to DAEs.

Let us assume that all the conditions of Theorem 4 are satisfied. Then the H.opf point is characterized (lccally) by the unicue solution of the following augmented system of nonlinear equations:

$$
\begin{aligned}
& 0=F(x, 0 ; \lambda) \\
& 0=\alpha A(x ; \lambda) r+B(x ; \lambda) s \\
& 0=\alpha A(x ; \lambda) s-B(x ; \lambda) r \\
& 0=N(r, s)
\end{aligned}
$$

where $N: \mathbb{R}^{n} \times \mathbb{R}^{n} \rightarrow \mathbb{R}^{2}$ is a normalization function and $A(x ; \lambda)=F_{p}(x, 0 ; \lambda)$, $B(x ; \lambda)=F_{x}(x, 0 ; \lambda)$. Following the same arguments as given in [12] and [20] for 
the ODE case, one can show that (13) has (locally) an isolated solution provided $N$ is chosen properly. For example, one could chose the following normalization function $N$ :

$$
\begin{aligned}
& 0=y^{t} r \\
& 0=r^{t} r-1
\end{aligned}
$$

with $y \in \mathbb{R}^{n}$ suitably selected [20].

Example 10. A nonlinear autonomous RLC circuit [4] which, for simplicity, is assumed to have a connected graph with $b$ branches can be described by the DAE

$$
\begin{aligned}
0 & =B v \\
0 & =Q i \\
q^{\prime} & =i_{c} \\
0 & =f_{c}\left(u_{c}, q\right) \\
\phi^{\prime} & =u_{l} \\
0 & =f_{l}\left(i_{l}, \phi\right) \\
0 & =f_{r}\left(u_{r}, i_{r}\right) .
\end{aligned}
$$

Here $v \in \mathbb{R}^{b}$ denotes the column vector of branch voltages and $i \in \mathbb{R}^{b}$ denotes the column vector of branch currents, partitioned, respectively, as $\left(v_{c}, v_{l}, v_{r}\right)$ and $\left(i_{c}, i_{l}, i_{r}\right)$ where subscript $c(l, r$, respectively) denotes the variable corresponding to capacitors (inductors, resistors, respectively). Equations (16) through (20) represent the constitute relations of the capacitors, inductors, and resistors. Equations (14) and (15) represent the interconnection of all circuit elements. The system of equations (14) through (20) is the standard system of circuit equations [4]; modifications of this system, such as the nodal analysis, are widely used in circuit analysis packages [4].

In [15], conditions for the existence of oscillations in oscillator circuits [4], such as the Wien bridge oscillator, were derived via a state-space analysis and the standard Hopf theorem for ODEs. The results in this paper show that the same results can be obtained by a direct analysis of the corresponding standard system of circuit equations, and a corresponding Hopf point can be computed by using (13). Such an approach is preferable especially for large circuits where the state-space form is difficult to obtain.

\section{Acknowledgments}

We would like to thank the anonymous referee for pointing out a simplification in the proof of Proposition 1. 


\section{References}

[1] R. Abraham, J. E. Marsden, and M. Ratiu, Manifolds, Tensor Analysis and Applications, Rearling, MA: Addison-Wesley, 1983.

[2] K. E. Brenan, S. L. Camptrell, and L. R. Petzold, The Numerical Solution of Initial Value Problems in Differential-Algebraic Equations, Amsterdan: North-Holland, 1989.

[3] S. L. Campbell, Solvability of General DAEs. North Carolina State University: Techn. Rzport NCSU NA.0292011, 1992.

[4] L. Chua, C. Desoer, and E. S. Kuh, Linear aitd Nonlinear Circuits, New York: McGraw Hill, 1987.

[5] L. Chua and A. Oka, Normal forms for constrained nonlinear differential equations, IEEE Trans. Circ. and Syst. 35(1988) 881-901.

[6] V. Dolezal, Practical stability criteria for sem state equations, Circ. Syst. Sig. Process. 6(1987), 3, 335-345.

[7] P. Van Dooren, The generalized eigenstructure problem in linear system theory, IEEE Trans. Automat. Control 26(19i31), 11-129.

[8] F. G. Gantmacher, Theo ry of Matrices, Vols. ] and II, New York: Chelsea, 1959.

[9] G. H. Golub and C. F. Van Loan, Matrix Computations, Baltimore, MD: The Johns Hopkins University Press, 1984.

[10] J. Guckenheimer and Pl. Holmes, Nonlinear Oscillations, Dynamical Systems and Bifurcations of Vector Fields, New York: Springer-Verlag, 1983.

[11] E. Hopf, Abzweigung siner periodischen Lösung von einer stationären Lösung eines Differentialsystems, Leipzig, Ber. Math.-Phys. Kl. Sächs. Akad. 94(1942), 303-322.

[12] A. D. Jepson, Numerical Hopf Bifurcation, Thesis, Part II, California Institute of Technology, Pasadena, 1981.

[13] R. März, On Quasilinear Index 2 Differential Algebraic Equations, Humboldt-Univ. Berlin, Techn. Report 269, 1991.

[14] N. H. McClamroch, Ffedback stabilization if control systems described by a class of nonlinear differential-algebraic equations, Systems Control Lett. 15(1990), 53-60.

[15] A. I. Mees and L. Chua, The Hopf bifurcation theorem and its application to nonlinear osc llations in circuits and systems, IEEE Trans. Cir. and Syst. 26(1979), 4, 235-254.

[16] P. J. Rabier and W. C. Rheinboldt, A generalized existence and uniqueness theorem for implicit differential-algebraic equations, J. Diff. and Integral Equations 4(1991), 681-694.

[17] P. J. Rabier and W. C. Rheinboldt, A geometric treatment of implicit DAEs, J. Diff. Equations, to appear.

[18] S. Reich, On a geometrical interpretation of differential-algebraic equations, Circ. syst. Sig. Process 9(1990), 4, 3547-382.

[19] W. C. Rheinboldt, Differential-algebraic systems as differential equations on manifolds, Math. Comp. 43(1984), 1473-1482.

[20] D. Roose and V. Hlavacek, A direct method for the computation of Hopf bifurcation points, SIAM J. Appl. Math. 45(19:35), 879-894.

[21] Q. Zheng, Hopf bifurcation in electonic c rcuits, In Mathematical Modelling and Simulation of Electronic Circuits and Semiconductor Devices (R. E. Bank, R. Bulirsch, and K. Meıten, eds.) pp. 44-61, Basel: Birkhäuser, 1990. 